\title{
Influence of nanocrystal size on the transport properties of Si nanocrystals
}

\author{
Xin Zhou, ${ }^{1, a)}$ Kouichi Usami, ${ }^{1}$ M. A. Rafiq, ${ }^{1, b)}$ Yoshishige Tsuchiya, ${ }^{1}$ Hiroshi Mizuta,,${ }^{1,2}$ and \\ Shunri Oda ${ }^{1}$ \\ ${ }^{1}$ Quantum Nanoelectronics Research Center, Department of Physical Electronics, Tokyo Institute \\ of Technology, O-Okayama, Meguro-ku, Tokyo 152-8552, Japan and SORST JST (Japan Science \\ and Technology), Japan \\ ${ }^{2}$ School of Electronics and Computer Science, University of Southampton, Highfield, \\ Southampton SO17 1BJ, United Kingdom
}

(Received 25 February 2008; accepted 29 April 2008; published online 30 July 2008)

\begin{abstract}
In this study, we have investigated the carrier transport mechanism across silicon nanocrystals with the $\mathrm{Al} / p-\mathrm{Si} / \mathrm{Si}$ nanocrystals/Al structure. Sizes of silicon nanocrystals were controlled at diameters of $\sim 6, \sim 8$, and $\sim 11 \mathrm{~nm}$. It is shown that the conductivity $\sigma$ of silicon nanocrystals, both as-grown and annealed, exhibits $\sigma \propto \exp \left[-\left(T_{0} / T\right)\right]^{1 / 2}$ behavior under low electrical fields and over a wide temperature range. The phenomenon of material constant $T_{0}$ increasing with the decrease of nanocrystal size has been observed. Considering nanocrystal size effect, experimental results can be explained by the hopping-percolation model. The influence of nanocrystal size on transport properties has been discussed. Based on this model, changes in $T_{0}$ after annealing treatment are attributed to an increase in effective decay length. (C) 2008 American Institute of Physics.
\end{abstract}

[DOI: $10.1063 / 1.2952036$ ]

\section{INTRODUCTION}

Semiconductor nanocrystals have attracted much interest in recent years due to their possible applications to Coulomb blockade devices, single electron transistors, and nonvolatile memories. ${ }^{1-3}$ In order to use nanocrystals for these applications, detailed knowledge of transport mechanisms across these nanocrystals becomes necessary and is considered indispensable. Due to the diversity of the nanocrystal system, various charge-transport mechanisms have been proposed. ${ }^{4}$ Among these are the $\mathrm{SiO}_{2}$-embedded $\mathrm{Si}$ nanocrystals, ${ }^{5} \mathrm{CdSe}$ nanocrystal ensembles, ${ }^{6}$ electrochemically doped $\mathrm{PbSe}$ nanocrystals, ${ }^{7}$ and Ge nanocrystals contained in thin films. ${ }^{8}$

Most research focusing on semiconductor nanocrystal ensembles or nanocrystals embedded in an insulator matrix usually had the problem of controlling size variation within a small range. This being the case, it is difficult to investigate the influence of nanocrystal size on the conductivity of these systems. In a former report, ${ }^{9}$ we demonstrated hopping conduction in size-controlled $\mathrm{Si}$ nanocrystals, $8 \pm 1 \mathrm{~nm}$ in size, from 40 to $200 \mathrm{~K}$. However, the effect of Si nanocrystals diameter variation on its transport properties still remains unexplained.

In this paper, we describe the effect of changing the diameter of Si nanocrystals in the thin layer of Si nanocrystals. In all samples with different nanocrystal sizes, we observed $\sigma \propto \exp \left[-\left(T_{0} / T\right)\right]^{1 / 2}$ dependence. This behavior can be explained well by the percolation-hopping conduction model. Based on this model, we discuss the influence of nanocrystal size on transport mechanism. We note that nano-

\footnotetext{
${ }^{a)}$ Electronic mail: xzhou@neo.pe.titech.ac.jp.

b) Also at National Centre for Nanotechnology, Pakistan Institute of Engineering and Applied Sciences, Islamabad, Pakistan.
}

crystal size plays a central role in the conduction process. In addition, the effect of annealing treatment has also been discussed.

\section{EXPERIMENTAL DETAILS}

Si nanocrystals were deposited on $p$-Si substrates with a resistivity of $0.02 \Omega \mathrm{cm}$ by very-high-frequency $\mathrm{SiH}_{4}$ plasma cell. ${ }^{10}$ Nanocrystal size was then varied by changing the flux of $\mathrm{SiH}_{4}$ while keeping the flux of $\mathrm{Ar}$ at $90 \mathrm{SCCM}$ (SCCM denotes cubic centimeter per minute at STP). The plasma power was controlled to be within 1.5-2 W. In this experiment, we prepared Si nanocrystals of diameters $6 \pm 1$, $8 \pm 1$, and $11 \pm 1 \mathrm{~nm}$ for each sample. After Si nanocrystal deposition, a thin $\mathrm{SiO}_{2}$ layer, about 1-2 $\mathrm{nm}$ in thickness, was deposited on the layer of nanocrystals by plasma-enhanced chemical vapor deposition. Because of the many voids in the nanocrystal film, this method prevents the unfavorable possibility of $\mathrm{Al}$ entering the nanocrystal film and touching the substrate, which can make the device short. Subsequently, electrodes that vary in size from $300 \times 300$ to 500 $\times 500 \mu \mathrm{m}^{2}$ were fabricated by $\mathrm{Al}$ evaporation through a metal mask. $\mathrm{Al} / p-\mathrm{Si} / \mathrm{Si}$ nanocrystals/Al device, a structure similar to that used in the previous study, ${ }^{9}$ was fabricated. Figure 1(a) illustrates a schematic diagram of the fabricated structure. Holes are injected into the nanocrystals by applying positive voltage on $p$-Si substrate. A scanning electron micrograph of these nanocrystals is shown in Fig. 1(b). The size of nanocrystals has been well controlled. Each dot is covered by a $\mathrm{SiO}_{2}$ shell $1.5 \mathrm{~nm}$ in thickness, which is formed by natural oxidation. Figure 1(c) demonstrates a crosssectional view of the Si nanocrystal layer. Prior to electrode fabrication, one sample from each group was annealed in hydrogen atmosphere at a temperature of $430{ }^{\circ} \mathrm{C}$. The detailed parameters of samples $\mathrm{A}, \mathrm{B}$, and $\mathrm{C}$ are listed in Table I. 

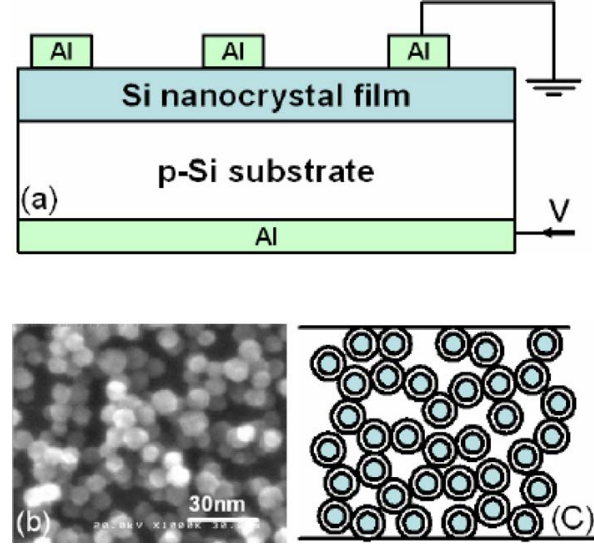

FIG. 1. (Color online) (a) Structure for the $I$ - $V$ measurements, (b) scanning electron micrograph of the Si nanocrystals, and (c) schematic cross-section view of Si nanocrystals.

\section{RESULTS AND DISCUSSION}

Figure 2 shows the current-voltage $(I-V)$ characteristics of the $\mathrm{Al} / p$-Si/Si nanocrystals/Al structure with different $\mathrm{Si}$ nanocrystal thicknesses and electrode sizes at $40 \mathrm{~K}$. Curves $\mathrm{A}$ and $\mathrm{C}$ represent the $I-V$ characteristics of samples $\mathrm{A}$ and $\mathrm{C}$, respectively, having an electrode size of $500 \times 500 \mathrm{\mu m}^{2}$. Curve B represents sample B having Si nanocrystals layer of $300 \mathrm{~nm}$ in thickness and $300 \times 300 \mu \mathrm{m}^{2}$ electrode size. All curves exhibit rectifying behavior. In order to confirm whether the rectifying behavior originates from the $\mathrm{Al} / p$-Si substrate or not, $I-V$ characteristics of the $\mathrm{Al} / p-\mathrm{Si}$ substrate/Al structure were measured at the same temperature, as shown in the inset of Fig. 2. The structure of $\mathrm{Al} / p-\mathrm{Si}$ substrate is found to make Ohmic contact as verified by the linearity of $I-V$ characteristics. The resistivity of the $\mathrm{Si}$ $p$-type substrate used in our device is $0.02 \Omega \mathrm{cm}$, which is low enough to avoid influence from the substrate. Therefore, we assumed that the rectifying behavior originates either from $\mathrm{Si}$ nanocrystals $/ p$-Si contact or $\mathrm{Al} / \mathrm{Si}$ nanocrystals interface.

As shown in Fig. 2, the $I-V$ characteristics do not scale with electrode size and reciprocal of the $\mathrm{Si}$ nanocrystal layer thickness. Both the voids in the nanocrystal film and the fluctuation of interface states make transport process much more complicated. In this kind of system, resistance is known to depend not only on the effective number of parallel current pathways $\left(N_{P}\right)$ under electrode, the number of tunnel junction $\left(N_{S}\right)$ in series, and the resistance of each junction $\left(R_{t}\right)$ but also on the distribution of $\mathrm{Si}$ nanocrystal in lateral and vertical directions. This is also clear from scanning electron microscopy (SEM) micrograph of nanocrystals pre-

TABLE I. Fabrication condition of Si nanocrystals.

\begin{tabular}{lcclc}
\hline \hline Sample & $\begin{array}{c}\text { Dot } \\
\text { diameter } \\
(\mathrm{nm})\end{array}$ & $\begin{array}{c}\text { Thickness } \\
(\mathrm{nm})\end{array}$ & $\begin{array}{l}\text { Electrode } \\
\text { size } \\
\left(\mu \mathrm{m}^{2}\right)\end{array}$ & $\begin{array}{c}\text { Annealing } \\
\text { temperature } \\
\left({ }^{\circ} \mathrm{C}\right)\end{array}$ \\
\hline A & $6 \pm 1$ & $\sim 150$ & $500 \times 500$ & 430 \\
B & $8 \pm 1$ & $\sim 300$ & $300 \times 300$ & 430 \\
C & $11 \pm 1$ & $\sim 150$ & $500 \times 500$ & 430 \\
\hline \hline
\end{tabular}

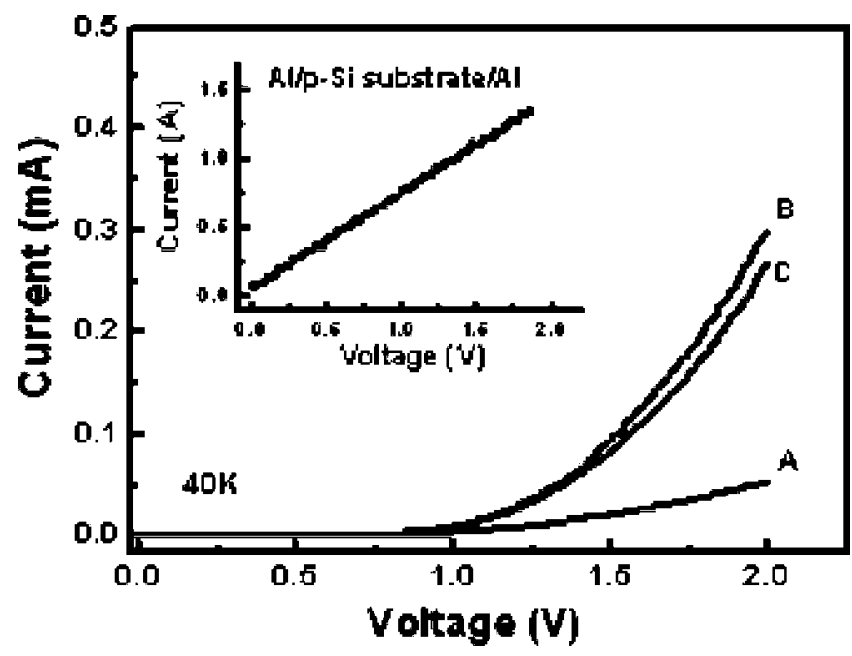

FIG. 2. $I-V$ characteristics of the $\mathrm{Al} / p-\mathrm{Si} / \mathrm{Si}$ nanocrystals/Al structure for samples $\mathrm{A}, \mathrm{B}$, and $\mathrm{C}$, measured at $40 \mathrm{~K}$. The inset figure shows the $I-V$ characteristics of the $\mathrm{Al} / p$-Si substrate/Al structure also measured at the same temperature.

sented in Fig. 1(b). Usually, $N_{P}$ and $R_{i}\left(R_{t}, N_{S}\right)$ scale with the density of $\mathrm{Si}$ nanocrystal in both vertical and lateral directions. With this in mind, we can describe it in simple formulas

$$
\begin{aligned}
& \frac{1}{R_{\mathrm{film}}}=\sum_{i=1}^{N_{P}} \frac{1}{R_{i}}, \\
& R_{i}=\sum_{t=1}^{N_{S}} R_{t},
\end{aligned}
$$

where $R_{i}$ is the total resistance of each current path. For samples with the same thickness, which contain the smaller dot, $R_{t}$ will be larger because of higher interface defects. Conversely, the effective number of both parallel current path $\left(N_{P}\right)$ and tunnel junction $\left(N_{S}\right)$ will be larger. Hence, the resistance of each sample will be mainly determined by the competition between $N_{P}$ and $R_{i}\left(R_{t}, N_{S}\right)$. Compared with the other two samples, sample B may have a larger number of effective parallel current paths playing a more important deciding role in terms of the whole nanocrystal film's resistance.

Figure 3 illustrates the $\ln (\sigma) \propto T^{-1 / 2}$ plots for samples B and $\mathrm{C}$ from 40 to $200 \mathrm{~K}$ and for sample A from 60 to $200 \mathrm{~K}$. The plots exhibit a linear behavior between $\ln (\sigma)$ and $T^{-1 / 2}$ for both as-grown and annealed samples. This indicates the same dependence of $\sigma \propto \exp \left[-\left(T_{0} / T\right)\right]^{1 / 2}$ similar to former reports. ${ }^{9}$ We have observed an inverse relationship in this plot, that is, the slope of the $\ln (\sigma) \propto T^{-1 / 2}$ plot increases as nanocrystal size decreases. This $\ln (\sigma) \propto T^{-1 / 2}$ behavior has been frequently explained by the Efros-Shklovskii variable range hopping (ES-VRH), percolation-hopping model, and the Sheng model. ${ }^{11-13}$ However, it has been determined that the ES-VRH model is not sufficient to explain reasonably the conduction mechanism in this kind of Si nanocrystal films. In relation to this, Sheng ${ }^{13}$ derived an $\ln (\sigma) \propto T^{-1 / 2}$ formula for granular metals. They proposed that $s$ and $d$ have some correlation. Based on that, $s E_{c}$ is supposed as a constant 

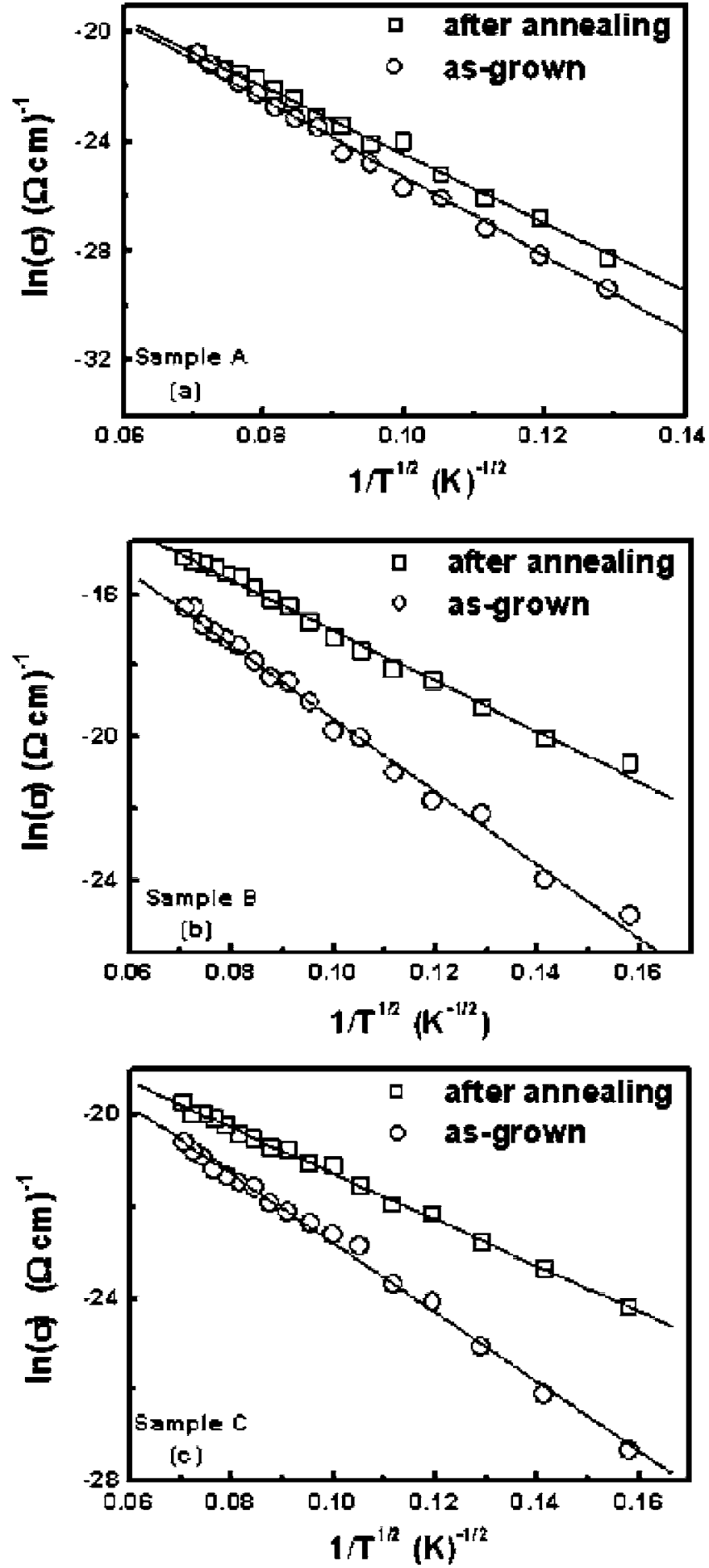

FIG. 3. $\ln (\sigma)$ as function of $T^{-1 / 2}$ for sample A measured from 60 to $200 \mathrm{~K}$ and samples B and C from 40 to $200 \mathrm{~K}$, as-grown and after hydrogen annealing. The solid lines are at least squares fits to the data.

whose value depends only on the composition of the sample and the dielectric constant of the insulator. Here, $s$ is the separation between grains, $d$ is grain size, and $E_{c}$ is charging energy. Thus, smaller $E_{c}$ usually is associated with larger tunneling barrier by this rule. For our samples, there is no correlation between nanocrystal separation and size since the $\mathrm{SiO}_{2}$ shell of each nanocrystal dot is caused by natural oxidation process. Therefore, to be able to quantitatively compare the experimental results with the theory, we used the percolation-hopping model in which $T_{0}$ is expressed by ${ }^{11}$
TABLE II. Estimated values of $\Delta E_{a \text {,max }}, T_{0}, \alpha$ and experimental value of $T_{0}$.

\begin{tabular}{lcccc}
\hline \hline Sample & $\begin{array}{c}\Delta E_{a, \max } \\
(\mathrm{meV})\end{array}$ & $T_{0}$ (Calc.) & $T_{0}$ (Expt.) & $\alpha$ (Calc.) \\
\hline $\mathrm{A}$ & 121 & $2.11 \times 10^{4}$ & $2.24 \times 10^{4}$ & 0.093 \\
$\mathrm{~B}$ & 62 & $1.07 \times 10^{4}$ & $1.04 \times 10^{4}$ & 0.103 \\
$\mathrm{C}$ & 32 & $5.22 \times 10^{3}$ & $5.33 \times 10^{3}$ & 0.105 \\
\hline \hline
\end{tabular}

$$
T_{0}=\frac{2 P_{c} s_{\max } E_{a, \max }}{k_{B} \alpha} .
$$

Here, $P_{c}$ is the percolation threshold, $s_{\max }$ is the maximum separation of particles in the current path, $k_{B}$ is the Boltzmann constant, and $\alpha$ is the effective decay length for the wave functions of carriers in the insulating region. According to this model, each nanocrystal semiconductor is characterized by (i) charging energy $E_{c}$ and (ii) $E_{d}$, which is the degree of the conduction band edge shift from the bulk edge due to size quantization. Hence, if a nanocrystal is surrounded by many nanocrystals, the charging energy of the nanocrystal is expressed as ${ }^{15}$

$$
E_{c}=\frac{e^{2}}{4 \pi \varepsilon \varepsilon_{0}} \frac{1}{d}\left[\frac{s / d}{0.5+s / d}\right] \text {. }
$$

The expression for $E_{d}$ is elucidated by ${ }^{16}$

$$
E_{d}=\frac{\hbar^{2} \pi^{2}}{2 m^{*}} \frac{1}{(d / 2)^{2}} .
$$

Here, the maximum value of the activation energy is considered as $E_{a, \text { max }}=E_{d, \text { max }}+\Delta E_{c} . E_{d, \text { max }}$ is the largest degree of conduction band edge shift. $\Delta E_{c}$ is the difference in charging energy between the large and small nanocrystals. $P_{c}$ is then chosen as 0.25 as an approximation corresponding to a simple cubic lattice. ${ }^{9}$ Employing the definition of nanocrystal separation as the distance between the edges of nanocrystals, the $s_{\max }$ in our sample is independent of dot size and can roughly be considered at a constant $\sim 3 \mathrm{~nm}$. Sheng et al. ${ }^{14}$ however, pointed out that the decay rate of electron wave function in the oxide can roughly be evaluated as $\chi$ $\approx \sqrt{2 m \Phi /(h / 2 \pi)^{2}}$, where $m$ denotes the carrier effective mass, $\Phi$ is the effective barrier height, and $h$ is the Planck constant. Using $\alpha=1 / \chi$ with an average hole effective mass of $0.57 \mathrm{~m}_{0}$ (Ref. 17) and a barrier height of $\Phi \sim 4.7 \mathrm{eV}$ (Ref. 18) for holes, $\alpha$ is approximately $0.1 \mathrm{~nm}$. Using the values of the above parameters, $T_{0}$ was calculated by Eq. (3) and the results are summarized in Table II. From the experimental results, we have observed that $T_{0}$ is independent of the nanocrystal layer thickness but is dependent on dot size. It is evident from Eq. (3) that $T_{0}$ is dependent on $s_{\max }, E_{a \text {,max }}$, and $\alpha$. For our samples, there is nearly no change in $s_{\max }$ when the size of nanocrystals decreases. Since $E_{c}$ and $E_{d}$ are functions of $d^{-1}$ and $d^{-2}$, respectively, the reduction of dot size makes significant increases in $E_{c}$ and $E_{d} . E_{a, \max }$ increases and becomes much more sensitive to the size of the nanocrystal as its size decreases. Therefore, $T_{0}$ increases as nanocrystal size decreases, which supports experimental results. As for the decay length, due to higher surface states and interface defects, the barrier height of the smaller nanocrystals is 
higher. Consequently, with a decrease in nanocrystal size, the effective decay length $\alpha$ for the holes in the $\mathrm{SiO}_{2}$ region usually becomes smaller. The measurement result of $T_{0}$ and Eq. (3) is used to calculate $\alpha$; the results are listed in Table II. We can easily perceive that $\alpha$ depends on the size of the nanocrystals.

After annealing treatment, the slope of the $\ln (\sigma) \propto T^{-1 / 2}$ plot for each sample decreases. The $T_{0}$ of samples A, B, and $\mathrm{C}$ changes to $1.54 \times 10^{4}, 4.97 \times 10^{3}$, and $2.53 \times 10^{3}$, respectively. This can be seen from the above discussion that change of $E_{a \text {,max }}$ and $\alpha$ can influence $T_{0}$. In order to determine the actual influence of hydrogen annealing treatment, some analyses based on Eq. (3) were performed. For sample $\mathrm{B}$, let us consider $\alpha \sim 0.1 \mathrm{~nm} . T_{0}$ (after annealing) is gained from Fig. 3. Then, Eq. (3) gives the value $E_{a, \max }=29 \mathrm{meV}$, as the value of $E_{a, \max }$ has a direct relationship with nanocrystal size. Thus, with the comparison between the $E_{a \text {, max }}$ of annealed sample B and as-grown sample $\mathrm{C}$, it is reasonable to conclude that the nanocrystal size, after annealing, should increase to be larger than 10-12 $\mathrm{nm}$. Verification of this change can be done by SEM. However, we did not find any change in the nanocrystal size after annealing treatment from SEM. Therefore, the change in slope is due to a decrease in $\alpha$, as indicated by Eq. (3). This is attributed to hydrogen annealing treatments that reduce the interface defects of $\mathrm{SiO}_{2} / \mathrm{Si}$, especially the dangling bonds, due to the $H$-passivation effect. This change of $\alpha$ can also be roughly evaluated by Eq. (3).

\section{CONCLUSION}

We have studied the transport mechanism across sizecontrolled Si nanocrystal films. We have investigated the influence of nanocrystal size on conduction mechanism.
Samples with different dot sizes exhibited $\ln (\sigma) \propto T^{-1 / 2}$ dependence. Slopes of $\ln (\sigma) \propto T^{-1 / 2}$ increase with the decrease in nanocrystal size. The $\ln (\sigma) \propto T^{-1 / 2}$ dependence can be explained by the percolation-hopping model. Using this model, the change in slopes of $\ln (\sigma) \propto T^{-1 / 2}$ curves after annealing treatment is associated with a change in $\alpha$.

\section{ACKNOWLEDGMENTS}

This work was partly supported by KAKENHI (Grant No. 19206035). M.A.R. is a JSPS postdoctoral fellow.

${ }^{1}$ Y. T. Tan, Z. A. K. Durrani, and H. Ahmed, J. Appl. Phys. 89, 1262 (2001).

${ }^{2}$ M. A. H. Khalafalla, H. Mizuta, and Z. A. K. Durrani, Phys. Rev. B 74, 035316 (2006)

${ }^{3}$ S. Tiwari, F. Rana, H. Hanafi, A. Hartstein, E. F. Crabbé, and K. Chan, Appl. Phys. Lett. 68, 1377 (1996).

${ }^{4}$ H. E. Romero and M. Drndic, Phys. Rev. Lett. 95, 156801 (2005).

${ }^{5}$ I. Balberg, E. Savir, J. Jedrzeijewski, A. G. Nassiopoulou, and S. Gardelis, Phys. Rev. B 75, 235329 (2007).

${ }^{6}$ D. Toker, I. Balberg, O. Zelaya-Angle, E. Savir, and O. Millo, Phys. Rev. B 73, 045317 (2006)

${ }^{7}$ B. L. Wehrenberg, D. Yu, J. S. Ma, and P. Guyot-Sinonnest, J. Phys. Chem. B 43, 109 (2005).

${ }^{8}$ S. Banerjee, S. Nozaki, and H. Morisaki, Appl. Phys. Lett. 76, 445 (2000). ${ }^{9}$ M. A. Rafiq, Y. Tsuchiya, H. Mizuta, S. Oda, S. Uno, Z. A. K. Durrani, and W. I. Milne, J. Appl. Phys. 100, 014303 (2006).

${ }^{10}$ T. Ifuku, M. Otobe, A. Itoh, and S. Oda, Jpn. J. Appl. Phys., Part 1 36, 4031 (1997).

${ }^{11}$ E. Šimánek, Solid State Commun. 40, 1021 (1981).

${ }^{12}$ A. L. Efros and B. I. Shklovaskii, J. Phys. C 8, L49 (1975).

${ }^{13}$ P. Sheng, Phys. Rev. Lett. 31, 44 (1973).

${ }^{14}$ P. Sheng and B. Abeles, Phys. Rev. Lett. 28, 34 (1972).

${ }^{15}$ B. Abeles, P. Sheng, M. D. Coutts, and Y. Arie, Adv. Phys. 24, 407 (1975).

${ }^{16}$ G.-F. Hohl, S. D. Baranovskii, J. A. Becker, F. Hensel, S. A. Quaiser, and M. T. Reetz, J. Appl. Phys. 78, 7130 (1995).

${ }^{17}$ R. A. Smith, Semiconductors, 2nd ed. (Cambridge University Press, Cambridge, 1978).

${ }^{18}$ T. Feng, G. Miller, and H. A. Atwater, J. Appl. Phys. 102, 034305 (2007). 